\title{
Exploring Societal Norms on Child Sexual Abuse in Albania: A Pilot Study
}

Enila Cenko, PhD

University of New York Tirana; enilacenko@unyt.edu.al

Klodiana Thartori

Children Today Center; klodthartori@gmail.com

Doi:10.5901/mjss.2016.v7n6p309

\section{Abstract}

Child sexual abuse is a complex public health problem with devastating outcomes for the child herself and the society at large. Decades of research attest to the high prevalence of these harmful acts toward children and their impact for overall individual and societal well-being. In Albania, a country with a relatively high young population, very little empirical research has been devoted to this issue, focusing mainly on the prevalence and incidence of the phenomenon. Furthermore, there is a paucity of data on societal and cultural norms contributing to the existence of child sexual abuse; whereas an in-depth understanding of these factors is necessary for any meaningful child protection interventions to take place. The current study attempts to offer helpful insights on societal norms surrounding child sexual abuse in Albania. It is a qualitative study, conducted in the region of Lushnja, involving discussions from focus groups with adult participants that are key figures in child protection; namely, parents, teachers and professionals working in the child protection area. Findings indicate that whereas child sexual abuse is perceived as being present in Albanian society, it remains hidden under a veil of secrecy due to the sensitive and taboo nature of the phenomenon. A thorough discussion of societal norms that contribute to the hidden nature of the phenomenon is provided along with an acknowledgment that there are protective factors within many Albanian families and society that should be strengthened and further developed in order to protect children.

Keywords: child sexual abuse, violence against children, societal norms, child protection, Albania

\section{Introduction}

Child abuse and neglect (CAN) constitutes a complex public health problem caused by numerous factors worldwide related to individual, family and community characteristics $(\mathrm{NIH}, 2007$; WHO, 1999). Child sexual abuse is one of the harmful acts children should be protected from. Still, many children do experience abuse. In regards to sexual abuse among children, an estimated 1 in 10 have experienced child sexual abuse (CSA) by the time they are 18 years of age (WHO, n.d.), while 1 in 5 women and 1 in 10 men report having been sexually abused as a child (WHO, 2014). Experts estimate that between 20 and 30 percent of all children and adolescents have been victims of sexual abuse at some time in their lives (Averdijk, Müller-Johnson \& Eisner, 2011).

Decades of research on the international sphere have addressed the issue of CSA; however, very little empirical research on the matter has been conducted in the Albania. Despite its spread and societal impact, the phenomenon of CSA in Albania continues to remain hidden; and, to date, official comprehensive country data on its prevalence and incidence are still lacking. Accurate estimates of its extent and characteristics in the general population are difficult to achieve mainly due to two reasons: a. the silence that surrounds maltreatment cases because of shame, social stigma and the consequent criminal liability leading to CAN underreporting and b. the lack of coordinated national CAN monitoring efforts that leads the majority of world countries to have neither valid nor reliable data on its magnitude. Especially concerning sexual abuse "fear, shame and loyalty prevent many victims from reporting the abuse."

Most of the existing data come from independent research conducted mainly by non-governmental organizations. The studies conducted so far have focused on the prevalence of the phenomenon of violence against children (VAC) in general, and no study to date has measured the prevalence and incidence of sexual abuse in Albania on its own. Furthermore, very little data is made available on the social and cultural norms associated with the phenomenon of sexual abuse, since the majority of the efforts to study the issue have focused on its epidemiology rather than on prevention and/or environmental factors.

One of the first studies to assess the magnitude of VAC in Albania (Kamani, Mato, \& Cangonji, 1997) indicates that 
the phenomenon is present, prevalent and tolerated in Albanian society. The occurrence of CSA, in particular, was also reported by participants. Out of the 609 participants, aged 12-17 years from different regions of Albania, about $9 \%$ of them reported that they had been involved against their will into acts of a sexual nature. Responses from 643 adult participants on their attitudes and beliefs toward child abuse in general indicate a distressing acceptance and tolerance of VAC, which is also often conceived as a necessary means of disciplining practices.

Another early study to report data on the prevalence of CSA in Albania was conducted in 2003 by the Multidisciplinary Centre for the Management of Child Maltreatment. The findings of this study indicate that $11 \%$ of the children involved in the study had experienced forms of sexual harassment, whereas $4 \%$ of the children reported experiencing sexual violence. This study also reiterates the previous finding that Albanian society is quite tolerant towards the use of violence against children as a way of controlling their behavior, stemming primarily from the patriarchal mentality prevalent in Albania.

Similar data were also reported by the study "Violence against Children in Albania", conducted by the Human Development Centre supported by UNICEF (Tamo \& Karaj, 2006). Involving responses of 1500 participants, including parents, teachers and children themselves, the findings of the study offer an additional testimony of the prevailing culture of VAC in Albania. In addition, $13.3 \%$ of the children involved in the study had experienced some form of sexual abuse, in different contexts, including the home, schools and institutions of care. Interestingly, this study indicated that the perpetrators of such abuse included family members, teachers, and caregivers in institutions of child care, community members, and strangers. Children involved in the study also report a general lack of information on issues related to child protection, and a recognized, immediate need to deal with this issue.

Data from the largest and most recent epidemiological study (Balkan Epidemiological Study on Child Abuse and Neglect) on VAC (Cenko, Hazizaj, Haxhiymeri, \& Coku, 2013) indicate high rates of different types of VAC (physical abuse, psychological abuse, sexual abuse and neglect) experienced by 11-, 13- and 16- year old children in Albania, both in terms of prevalence and incidence, indicating ongoing victimization. The study focused on VAC in the context of the home and utilized the ICAST questionnaires adapted for use in the Albanian context. Findings on data collected from 3328 children, both in rural and urban areas of Albania, indicated that $11.4 \%$ and $4.88 \%$ of the children had experienced sexual violence and contact sexual violence respectively during their lifetime. The incidence for sexual abuse in children (that is the occurrence of such cases in the year preceding data collection) was $9.15 \%$ and $4.10 \%$ of children for sexual violence and contact sexual violence respectively.

Another study conducted under the larger framework of the BECAN project, the BECAN Case based Surveillance study (Hazizaj, Coku, Cenko \& Haxhiymeri, 2013) provides findings on the incidence of all forms of CAN based on data collected by existing archives and databases of agencies and facilities involved in the handling of CAN cases, such as child protection services, health, judicial and police services and NGOs. The study reports a striking difference in terms of reported inappropriate sexual behavior between boys and girls, with the latest reporting the highest rate. About 7 per cent of agencies report that children exhibit inappropriate sexual behavior related to their age, with girls counting for almost 12 per cent, while boys (all age groups) are reported only at the rate of 2 per cent. This difference indicates that girls aged between 11-13-16 years of age have been exposed to a higher degree of sexual violence compared to boys of the same age.

These aforementioned studies as well as other earlier and smaller-scale assessments indicate that the phenomenon of VAC in Albania is highly prevalent in Albanian society, and that it is accompanied by widespread acceptance of VAC as disciplinary means of raising children. Despite the outcomes outlined by this body of research, what remains to be explored are the societal and contextually relevant factors that contribute to the phenomenon perpetuating itself over time. Previous research suggests that VAC in Albania has deep seated roots in the patriarchal traditions characterized by parental authority, adherence to an honor-and-shame system, and customs of hierarchal ordering with the family and the intergenerational family (see Harr \& Dhamo, 2009; Haxhiymeri, Kulluri \& Hazizaj, 2005). To date, however, very little research has been conducted on the environmental factors that create and maintain the phenomenon. A thorough understanding of these factors, including an assessment of social attitudes, beliefs, and perceptions, is necessary in order to be able to take action towards the prevention of CSA. In this context, there is an immediate need to explore the perceptions, attitudes, and beliefs of Albanian people towards children in general and child abuse in particular.

Several questions are of importance in exploring the societal and cultural norms that contribute to the existence of CSA in the Albanian context. For instance, are there social norms, which contribute to the prevalence of CSA in Albania? What are the attitudes of the community that aid in keeping the phenomenon hidden? Are members of the community equipped with the adequate knowledge on CSA, which is necessary in order to prevent the phenomenon and ensure the protection of children? 
The current study attempts to address this existing gap in the literature and understanding of CSA in Albania, by focusing on an exploration of the societal and cultural norms toward CSA in the Albanian context. It aims to understand how CSA is perceived in the community of the region of Lushnja, what are the attitudes toward the phenomenon which may contribute to the issue and that influence the social norms surrounding CSA, what is the current knowledge of this form of child abuse in this community; and what are the perceptions on why the phenomenon continues to remain hidden and often unreported. More specifically, the research questions guiding the present study are as follows: What are the prevalent attitudes of adults towards CSA in Albania? Is this phenomenon publicly accepted or rejected; is it implicitly tolerated? What are some societal and cultural norms that may contribute to the occurrence and maintenance of the phenomenon? Related to this question, what are the societal perceptions on what constitutes childhood and when is an individual ready for sexual activity? What are the current knowledge and perceptions on how prevalent CSA is in Albania? What are the perceptions and knowledge of adult caregivers of children on what constitutes CSA and what are the forms in which it occurs? How do adult caregivers communicate with children about CSA and its prevention? Does such communication exist? How often is CSA reported and what are some of the factors that contribute to lack of reporting and, hence, in maintaining the phenomenon hidden?

\section{Methods}

The study was conducted at a pilot level in the region of Lushnja, during the period of December 2015-January 2016. It was a qualitative study, consisting of data collected via focus group discussions led with parents and teachers of children of ages 11-15 and 16-18, and professionals that work in the area of child protection. Qualitative methods were used for this study primarily because the aim of the study was to lead to an in-depth understanding of perceptions, attitudes, and knowledge of participants on CSA, rather than a large assessment of the phenomenon.

\subsection{Participants}

A total of 9 focus groups with 95 participants were conducted in urban and rural parts of Lushnja, including Lushnja city and the rural areas of Çermë and Fjershegan. The representation of participants from both urban and rural areas is important for a comprehensive understanding of the perceptions, attitudes, and knowledge on CSA, given the differences in social norms that characterize urban and rural areas in Albania in general. A purposive sampling method was used for the selection of participants. The focus group with professionals working in the area of child protection included 5 social workers, 4 psychologists, and 2 educational specialists (7 of them working in Child Protection Units as child protection specialists and 4 working in school settings as school psychologists). The majority of participants in the focus groups were female. More specific information on age and gender characteristics of participants is outlined in Table 1 below.

Table 1: Demographic characteristics of participants of focus groups

\begin{tabular}{|c|c|c|c|}
\hline & Teachers & Parents & Professionals \\
\hline $\begin{array}{l}\text { Urban area } \\
\text { Grades 5-9 }\end{array}$ & $\begin{array}{l}1 \text { focus group } \\
10 \text { participants } \\
\text { Age: mean } 40 \text { years } \\
\text { Range } 27-50 \text { years } \\
6 \text { female } \\
4 \text { male }\end{array}$ & $\begin{array}{l}1 \text { focus group } \\
15 \text { participants } \\
\text { Age: mean } 39 \text { years } \\
\text { Range } 35-47 \text { years } \\
15 \text { female }\end{array}$ & \\
\hline $\begin{array}{l}\text { Rural area } \\
\text { Grades 5-9 }\end{array}$ & $\begin{array}{l}1 \text { focus group } \\
12 \text { participants } \\
\text { Age: mean } 39 \text { years } \\
\text { Range } 26-48 \text { years } \\
9 \text { female } \\
3 \text { male }\end{array}$ & $\begin{array}{l}1 \text { focus group } \\
12 \text { participants } \\
\text { Age: mean } 41 \text { years } \\
\text { Range } 32-52 \text { years } \\
12 \text { female }\end{array}$ & \\
\hline $\begin{array}{l}\text { Urban area } \\
\text { Grades 10-12 }\end{array}$ & $\begin{array}{l}1 \text { focus group } \\
7 \text { participants } \\
\text { Age: mean } 38 \text { years } \\
\text { Range } 27-50 \text { years } \\
5 \text { female } \\
2 \text { male }\end{array}$ & $\begin{array}{l}1 \text { focus group } \\
9 \text { participants } \\
\text { Age: mean } 45 \text { years } \\
\text { Range } 39-52 \text { years } \\
8 \text { female } \\
1 \text { male }\end{array}$ & \\
\hline Rural area & 1 focus group & 1 focus group & \\
\hline
\end{tabular}




\begin{tabular}{|l|l|l|l|}
\hline Grades 10-12 & $\begin{array}{l}9 \text { participants } \\
\text { Age: mean 36 years } \\
\text { Range 24-44 years } \\
5 \text { female } \\
4 \text { male }\end{array}$ & $\begin{array}{l}11 \text { participants } \\
\text { Age: mean 41 years } \\
\text { Range 34-48 years } \\
11 \text { female }\end{array}$ & \\
\hline & & & $\begin{array}{l}1 \text { focus group } \\
11 \text { participants } \\
\text { Age: mean 34 years } \\
\text { Range 25-46 years } \\
6 \text { female } \\
\text { male }\end{array}$ \\
\hline
\end{tabular}

\subsection{Procedure}

The topics that were discussed in focus groups explored people's perceptions, attitudes, and knowledge on CSA in general, and more specifically views about definitions of abuse, their conceptualization of childhood, their knowledge of the phenomenon, their reflections on prevalence, and societal norms that contribute to public invisibility of the issue. Examples of questions asked to focus group participants included: "What is the age when a person stops being a child?", "When do you think a person is ready for sexual activity?", "What types of behavior do you think constitute CSA?" and so on. Focus groups were led by trained facilitators with the aim to encourage participants to provide honest responses and to lead to in-depth understanding of their perceptions, attitudes and beliefs. Each focus group lasted for about one hour and half and participants' responses were audio-recorded. An assistant facilitator also kept detailed noted on the focus group discussions as well as non-verbal elements of communication during the course of discussions.

\subsection{Data analysis}

All focus group discussions were transcribed and transcriptions were checked for uniformity and accuracy. Generic themes were constructed based on the research questions, including perceptions, attitudes and beliefs on the concept of childhood; perceptions and attitudes on the phenomenon of CSA and the socio-cultural norms associated with it; perceptions and knowledge on what constitutes CSA; perceptions on the scale of the problem and its prevalence in the community; perceptions and attitudes on the need for communication with children on CSA; and perceptions and attitudes on the level of reporting of cases of CSA. Both classical content analysis and constant comparison analysis to see what themes emerged during discussions, in order to assess whether themes that emerged from one group were also present in other groups as well.

\subsection{Ethical standards}

Research was conducted based on clear ethical standards which assured confidentiality, privacy, anonymity and informed consent. All participants were asked to give oral consent for participation in the focus group discussions and the research team was trained on the safeguarding of ethical issues.

\section{Discussion of Findings}

The findings presented below are generated by the discussions that took place in focus groups with parents, teachers and professionals, and are presented in terms of the general themes generated by the research questions of the study. These findings aim to shed light towards an in-depth understanding of the perceptions, attitudes and knowledge on CSA in the region of Lushnja, rather than provide generalized findings about the Albanian context at large. The main findings are grouped based on the major themes that emerged in all conducted focus groups.

\subsection{Participants' perceptions and attitudes on the phenomenon of CSA and the socio-cultural norms associated with it.}

It was evident that CSA is recognized by all as a distressing phenomenon that is clearly present in Albanian society. Participants also identified an immediate need to speak openly about and discuss the phenomenon at the community level in order to take concrete steps in ensuring the protection of children. As one participant noted in highlighting the importance of discussing the phenomenon of CSA: 
"It is a 100\% important; very important in all directions: for the today, for tomorrow, for everything, for the only treasure that we Albanians have, that is the children. We have no other treasures."

In addition to discussing about CSA publicly and openly, another immediate need that was identified was to speak with children themselves about CSA and how to protect themselves from it. As one participant put it:

"We must talk to children about this issue, we must inform them accordingly in order to prepare them, to protect them. It is best if I speak to my child about sexual abuse, rather than her getting information from elsewhere, since that other information may be the wrong one."

The need to address the phenomenon publicly and to involve children in this public discourse becomes highly relevant and takes a special meaning when considering an important theme that emerged from the discussions - that of keeping CSA under a veil of secrecy due to several social and cultural norms that are prevalent in Albania. One such norm that was repeatedly identified during the discussions is the existing taboos in Albanian mentality in relation to sexual activity in general, and especially with regards to sexual activity prior or outside of marriage. The following comment of a parent captures this idea that was expressed often times:

"Albanian mentality, especially in rural areas, cannot grasp the occurence of sexual relations outside of marriage. I, for example, cannot imagine my daughter [engaging in sexual activity] without being official [i.e. being married]."

This socio-cultural norm of preserving honor and refraining from sexual activity until marriage is especially prevalent for females due to the patriarchal mentality that is still present in many areas of Albania, especially rural ones. This theme of placing special emphasis on girls rather than boys in terms of delaying sexual activity until marriage was emphasized several times during the discussions.

Another social norm that contributes to the keeping CSA hidden as a problem is associated with the widespread acceptance of violence in general, and of VAC in particular in Albanian society. This societal and cultural tolerance of violence desensitizes community members towards cases of VAC, and hinders adequate steps and actions in identifying, reporting, preventing, and protecting children from violence in general as well as sexual violence and abuse. As was noted by one participant:

\begin{abstract}
"Sexual abuse is not accepted by our society, but other forms of violence such as physical and psychological abuse are accepted by our culture. Their presence is disconcerting, these types of violence are widely practiced in schools, families, and in the community.

The Albanian family in general, and families in this community, are violent, whether we like it or not. Parents engage their children without asking for their consent. This is not the only case of violence. I tell you another type: The parent comes and sees me to ask how the child is doing in school, how he is behaving. They say: "If you notice something wrong, hit him." This is a reflection of violence. Forced engagements too. These are indicators that violence is widely accepted by our society."
\end{abstract}

The majority of participants acknowledged that these existing norms are contributing factors to an inadequate treatment of the problem, and there is an urgent need to overcome them in order to ensure the protection of children from abuse and violence of all forms.

\title{
3.2 Perceptions and knowledge on what constitutes CSA
}

Participants' comments on the question of what is CSA reflect a range of perceptions and levels of knowledge of what constitutes CSA. There was consensus among participants that some types of behaviors, such as rape or violent intercourse with a minor or incest constitute CSA. Other main forms of CSA that were identified by the majority of respondents included sexualized touching, exposing children to pornography or adult sexual behaviors, and inappropriate verbal advances by older men towards minors. However, respondents differed in their views about other forms of abuse.

At the other hand, there were several participants who disagreed with the perception of the aforementioned behaviors as constituting CSA and suggested that these types of behavior are morally wrong, but do not necessarily constitute sexual abuse.

Researchers also noticed that often times CSA was blurred in the minds of the participants with consensual sexual activity that occurs among adolescents and/or with homosexual activity. These perceptions are captured in the comment of one participant, who states: 
"When we speak about abuse of course we mean extreme cases like an adult taking a child to bed as was the case of a teacher that was doing that with his pupils. This case happened in our community and that is extreme. But on the other hand, abuse can also happen among adolescents in high school, when they hug and touch each other. Because during puberty, cases like this happen. Or cases of boys with boys and girls with girls. We are hearing all sorts of these things nowadays."

These perceptions are linked with the socio-cultural norms where any type of sexual activity before marriage as well as homosexuality are deemed socially unacceptable, thus, leading to the shunning of adolescents and adolescents from engaging in any type of sexualized behavior outside of the context of marriage. In itself, these norms further contribute to the hidden and taboo nature of CSA, rather than towards its prevention.

\subsection{Perceptions and attitudes on the construction of childhood}

In order to undertand perceptions and attitudes toward CSA, it is essential to understand what are the various ways of defining childhood. Participants were asked during the focus group discussions, who they considered to be a child, and what would be the appropriate age to initiate into sexual activity. A range of responses were given to these questions, with the majority of participants agreeing that childhood ends at 18 , and that this is also the most appropriate time to commence sexual activity.

It is interesting to note that in the range of responses reflecting perceptions and attitudes on childhood, a number of participants expressed that a person is ready for sexual activity as early as the onset of puberty and more specifically at the age of $12-13$ years old. Participants also gave anecdotal evidence of children adopting sexualized behavior at an earlier age than before, mainly due to the influence of media and the internet, which model sexualized behavior and present them as norms, which become attractive to the younger generation. One participant said that:

"The age in which children start having sex has decreased. If you look carefully, as early as 7th grade, children use sexualized vocabulary and engage in inappropriate and prohibited acts that they adopt from what they see on TV. Nowadays, it is very difficult to control children, due to the internet and them having cell phones becoming a phenomenon."

The general perceptions and attitudes on the right age to commence sexual activity were different for boys and girls, with boys perceived as being entitled to enter sexual activity at any age, whereas girls will need to wait until marriage. These perceptions and attitudes reflect the prevalent patriarchal mentality in Albanian society in general, as reflected in the comment below:

"Who has children that are boys says "it's OK, I have a son", whereas who has daughters has a different situation, a more difficult job. As a parent, I keep the son under my control until 18, and after that I tell him "you look after yourself."

There was also a difference between rural and urban areas in terms of considering what is the appropriate age for sexual activity, with participants from rural areas denoting an earlier age than participants from urban areas. Thus, as expressed in the following comment:

"In our village girls get engaged early, once they finish 9th grade or the first year of high school, they get engaged. In some cases, parents engage them even when they are in the 7th grade."

Early age engagement and marriage for females is an existing practice in Albania, especially in rural areas. It is related to the role of females in society, seen as second order citizens, whom need to be married off, otherwise they are a burden for their families. Even thought the legal age for an individual to enter marriage is 18 years old (and in special cases, with court permission at 16 years of age), often times females are engaged at a much earlier age (14 or 15 years old). They are engaged or married at an early age to ensure their virginity and honor. One participant expressed her concern about the way girls are raised in Albanian rural communities:

"The Albanian family is driven to raise girls with this mentality: "You are of a certain age, I need to find you a husband now, you are a burden for the family."

The phenomenon of underage engagement/marriage was perceived to be a common occurence, particularly in rural areas. Despite the widespread acceptance of the phenomenon in the community, which does not consider these 
cases as CSA, several participants in the focus expressed that this practice should be condemned as it leads directly or indirectly to CSA. This finding is interesting per se, since it indicates that even though the phenomenon of early engagement/marriage is present in Albanian society (especially in rural and Roma communities), the general attitude towards the phenomenon is that it is morally wrong, linked with CSA, and detrimental to the child's well-being.

\subsection{Perceptions on the scale of CSA and its prevalence in the community}

Discussions on how often CSA occurs in their own community was initially met with a tendency toward denial in focus groups involving parents and teachers. As focus groups advanced, there was an acknowledgement that CSA occurs in the respective communities as well. Parents and teachers relied mainly on anecdotal evidence or cases highly publicized by the media to illustrate the phenomenon. They also acknowledged that if CSA happens it is kept under strict secrecy due to the existing socio-cultural norms. As one participant commented:

"Only rarely do people who show or report such cases. It's the mentality that prohibits them from doing so. Even if such a case happens, the family of the child tries to deal with it on its own. They can even kill the person who did, but nothing should be found out by others that abuse happened."

The reluctance to accept that cases of CSA happen in their own communities is related not so much on the lack of information on these cases, but rather on the secrecy surrounding the phenomenon as illustrated in the last comment above. On the other hand, there are parents and teachers who are willing to admit that CSA occurs in their communities and to their families.

Professionals working in the area of child protection in the region of Lushnja also attest that CSA is an ever present phenomenon in this community. In fact, the perceptions of professionals sustained by their immediate contact with cases of this nature is that CSA is "a real presence in the community", it happens at a "large scale" and that "children are left unprotected." Professionals also recognize the fact the phenomenon remains largely hidden due to the norms that are associated with it. Thus, the only occasions when CSA is reported is when the cases are sever and cannot be neglected. In fact, they report that:

"We only come to know of CSA when the cases are very severe. These are the only cases that come to us. Lighter forms of sexual abuse are never acknowledged, they are never "perceived as such, they are not a big deal."

Independently of participants' perceptions of the prevalence and scale of the problem of CSA in their own communities, the main view expressed by them is that serious measures should be taken to prevent CSA cases. These measures should be taken at the level of the family, school, community and state.

\subsection{Perceptions and attitudes on the need for communication with children on CSA}

During the focus group discussions, participants were asked on their perceptions of how well children are informed in terms of CSA and of protecting themselves. The general consensus was that children do not have the necessary and sufficient information to protect themselves. In the cases when they have access to information, it is through their friends, peers, the media, and the internet. These sources of information are deemed by parents, teachers and professionals to be the wrong sources of information, since they may increase the risk of children being exposed to CSA, as is depicted in the comments below:

"Children today get most of the information from the books, friends and the internet. But one must be really careful in terms of what information children get because friends can take the child down the wrong path. Like the case of two girls in one neighborhood. The older one was taking the younger to seek out boys. This is abuse I think."

It was generally expressed as a common attitude that families and schools need to educate their children and engage into open discussions with them about sexual activity in general. Sex should not be considered taboo. As one mother put it:

"The more you speak with the child about risk [of sexual abuse], the more you make him think before he acts, the more you protect him."

Despite expressing this common need of establishing an open communication with the children and changing 
social norms regarding the discussion of CSA and sexual activity in general, parents and teachers identified that this open communication rarely occurs. Apparently, the social norms of considering sex as a taboo and that contribute to the culture of silence surrounding CSA, also make discussions between parents and children as well as teachers and children very difficult to take place. These socio-cultural norms are especially prevalent in rural areas. A parent in the urban area commented:

"The Albanian family has a closed and old mentality. Of course that it is important to give information [about sexual activity and abuse] to children, but most of the times it is very difficult to have these conversations with them. My children may know lot about these topic, but I, as a mother, cannot imagine talking to them about this."

Part of the reason how these socio-cultural norms hinder the process of open dialogue with regards to sexuality was also the belief that if one speaks to children about sex, this may actually encourage children to have sex. This popular view was apparently prevalent, particulary in rural communities, as evidenced by the comment below:

"We are afraid to talk to children [about sex] because we are afraid that maybe we push them towards having sex when they may have not been thinking about it at all."

The role of the school was deemed as essential in this process of informing children on sexuality in general and how to protect themselves from CSA. There was a discrepancy between the responses of teachers in urban and rural areas of how well this process was done in schools. Teachers from urban areas had a greater inclination that sexual education took place in their schools and that children received information on how to protect themselves and prevent CSA. In rural areas, however, teachers reported that sexual education does not take place, not even in high schools, either because it is deemed unneccessary or because it is very difficult to implement due to the socio-cultural norms that prevent the open discussion of sexuality in these communities. In most cases, teachers report that due to the aforementioned reasons, they skip sexual education altogether, even though it is part of the school curricula.

Researchers noted, throughout the focus group discussions, that parents and teachers tended to emphasize the role that each of party should play in communicating children about sexuality and CSA. Even though the widespread acceptance was that education of the children was primarily the role of the family, parents seemed to expect more than what is being currently done by the school in this direction. One parent said:

"Sexual education classes must be included in the curricula so that children are no longer surprised when we speak to them, at home, about sexual abuse. In this way, we have an easier time talking to them about this topic."

On the other hand, teachers clearly expressed the immediate need for parents to take responsibility on the matter, to break through the social norms that impede the open communication with children, to become more educated on matters of CSA and to be more involved in their children's lives. At this point, however, it is clear that there is an immediate need to increase the communication flow with children in terms of CSA, since parents, teachers, and professionals all recognize that this communication is done at minimal levels. Unless concrete steps are taken to change this situation, children are left vulnerable to CSA.

\subsection{Perceptions and attitudes on the level of reporting of cases of CSA}

Children should be able to disclose instances of sexual abuse to trusting adults. However, findings from the discussions suggest that most cases of CSA are not reported to caregivers and even less to authorities. Instead, children seem to rely on same-age friends to share this sort of information. Even when the child discloses abuse to family or another caregiver such as the teacher, abuse is not always reported both by the family as well as other community members.

One of the main reasons why CSA is reported at minimal levels is associated with the socio-cultural norms that associate it, according to which it is best to keep the knowledge of abuse within the family rather than risk destroying the reputation and "the good name" of the family. The following comment illustrates these types of attitudes:

"I think that if it [sexual abuse] happens, it should be kept within the family. There is no need for other people to know. We are a family with good reputation. We are concerned that if something like this happens, the aunt should not find out, others should not find out. Better keep it within the family. As they say, it is better to lose one eye than lose one's reputation."

Unfortunately, these attitudes create conditions that even when parents come to know of abuse of their own 
children, they choose to not report it for fear of losing face in the community. In this way, these prevailing social norms create conditions that facilitate and maintain CSA. This is especially the case in situations where CSA occurs within the family, thus leaving children exposed to risk.

Members of the community are also often reluctant to report abuse when they come across it. There is also fear of the consequences of reporting, including professionals and teachers, since there is concern that someone reporting abuse could be identified by others in the community and suffer as a consequence, as illustrated in the comment below.

"It is because of the fear of what may happen if you report. This is a small community, heavily influnced by the opinion of others. If you report the child, the parent may become aggressive towards you, may become your enemy. The parent may say: "You ruined the reputation of my daughter. Who are you to protect my child? She should have come to me first." This is difficult when the child has reported any type of abuse, but especially difficult in cases of sexual abuse."

Due to the widespread culture of violence as a means of solving problems and of the lack of confidentiality in small communities, the reluctance to report is also directly related with fear of violent consequences of reporting. Relying on self-justice rather than reporting CSA, also contributes to maintaining the phenomenon hidden. This choice is due to the need to keep the reputation of the family as well as lack of trust in competent authorities and institutions to deal with the issue.

The mistrust of children due to their lower status in society also leads to not taking their claims seriously and therefore not reporting abuse when it occurs. As such, it was expressed that before reporting abuse to the authorities, one needs to search for the veracity of the child's word. The following comment illustrates how the testimony of children is not taken seriously, thus, allowing abuse to perpetuate:

"There was this case in my neighborhood of a teacher abusing with a 13 year old girl. The abuse came out but the teacher continued to work at the school until he retired. It is a matter of not believing the word of the child. It happens a lot unfortunately."

Other reasons identified as associated with lack of or underreporting of cases of CSA have to do with a general perception of the child protection system and legislative measures as incomplete or inadequate in ensuring the appropriate actions in protecting children. The general perception is that people fail to report due to a mistrust in the implementation of laws that protect children and put in place punitive measures for perpetrators. One example given during the discussions is:

"In our community there are people who mentally sick hanging out in public spaces around children. When he gets caught doing something wrong, the law declares him insane, and he is declared not guilty."

\section{Conclusions}

The current study provides important insights and in-depth understanding of the social and cultural norms that surround CSA in the Albanian society. Findings show that members of the community are aware of the existence of the phenomenon, condemn it and express the need to be proactive at an individual, community, and state level in order to protect children. The study also shows that while child abuse is not a new phenomenon there are some factors contributing to it such as the culture of secrecy and the perpetuation of the issue. The understanding of these factors and norms is essential before large scale interventions can take place and child protection measures can be effectively taken. Furthermore, the findings from the discussions indicate that there are protective factors within many Albanian families and society that should be strengthened and further developed in order to protect children.

\section{References}

Averdijk, M., Müller-Johnson, K., \& Eisner, M. (2011). Sexual victimization of children and adolescents in Switzerland. Zurich, Switzerland: UBS Optimus Foundation. Available at: www.optimusstudy.org/fileadmin/user_upload/documents/Full_Report_ Schweiz/Optimus Study Final_Report 2012 e.pdf. Last retrieved on May 7, 2016.

Cenko, E. Haxhiymeri, E., Hazizaj, A. \& Coku, B. (2013). Report on Incidence and Prevalence rates, types and determinants of CAN (on national and Balkan level) in children 11, 13 and 16 years old. CRCA, Albania. [Online]. Available at www.becan.eu/node29. Last retrieved on August 25, 2016.

Harr, R., \& Dhamo, M. (2009): Domestic violence in Albania: A national population-based survey. Tirana, Albania: National Institute of Statistics, Albania. 
Hazizaj, A., Coku, B, Cenko, E. \& Haxhiymeri, E. (2013).Albania Case-based surveillance study on violence against children. CRCA Albania. DOl: 10.13140/2.1.3608.544

Haxhiymeri, E., Kulluri, E., \& Hazizaj, A. (2005). Child abuse in the Albanian family. Tirana, Albania: Children's Human Rights Centre of Albania.

Kamani, P., Mato, E., \& Cangonji, E. (1997). The child abuse phenomenon in the Albanian society. Tirana, Albania: Refleksione.

Multidisciplinary Centre for the Management of Child Maltreatment (MCMCM) (2000). The situation of child maltreatment phenomenon in school and family environment in Tirana. Tirana, Albania: Soros Foundation

National Institutes of Health (NIH) (2007). Research on Interventions for Child Abuse and Neglect (R01) Program. [Online]. Available at http://grants.nih.gov/grants/guide/pa-files/pa-07-437.html_Last retrieved on May 10, 2016.

Tamo, A., \& Karaj, Th. (2006).Violence against Children in Albania. Tirana: Human Development Centre and UNICEF.

World Health Organization (1999). Report of the consultation on child abuse prevention. WHO, Geneva, 29-31.

WHO (n.d.) Regional consultation to develop WHO action plan on interpersonal violence. [Online] Available at www.euro.who.int/en/ mediacentre/events/events/2015/05/regional-consultation-to-develop-who-action-plan-on-interpersonal-violence__Last retrieved on June 20, 2016.

WHO (2014).Global status report on violence prevention. Geneva: World Health Organization. 